\title{
Single-Cell Transcriptome Profiling Reveals Intratumoral Heterogeneity in Human Chordomas
}

\section{Wanru Duan}

Xuanwu Hospital Department of Neurosurgery

\section{Boyan Zhang}

Xuanwu Hospital

\section{Xinqiang Li}

The Affiliated Hospital of Qingdao University

\section{Shanhang Jia}

Xuanwu Hospital Department of Neurosurgery

\section{Zong Xin}

Xuanwu Hospital Department of Neurosurgery

\section{Qiang Jian}

Xuanwu Hospital Department of Neurosurgery

\section{Fengzeng Jian}

Xuanwu Hospital Department of Neurosurgery

\section{Dean Chou}

University of California San Francisco Department of Neurology

\section{Zan Chen ( $\nabla$ chenzan66@163.com)}

\author{
Xuanwu Hospital
}

\section{Research}

Keywords: Chordoma, Single-cell RNA sequencing, transcriptomic, TGF- $\beta$ signaling pathway, tumor microenvironment, tumor immunity

Posted Date: February 4th, 2021

DOI: https://doi.org/10.21203/rs.3.rs-167938/v1

License: (c) (i) This work is licensed under a Creative Commons Attribution 4.0 International License. Read Full License 


\section{Abstract}

Background: Chordoma is a rare and aggressive bone tumor with high recurrence. The intra-tumoral heterogeneity of chordomas is poorly understood, limiting the development of effective therapeutic strategies.

Methods: Single-cell RNA sequencing was performed to delineate the transcriptomic landscape of chordomas. Six tumor samples of pathologically classical chordomas were obtained, and 33,737 cells that passed quality control were included in the analysis.

Results: The main cellular populations identified with specific markers were: chordomas cells (6392, 47.6\%), fibroblasts (6945, 20.6\%), mononuclear phagocytes (4734, 14.0\%), and T/NK cells (3944, 11.7\%). Downstream analysis was performed according to each cellular population. There were six subclusters of chordomas, which exhibited properties of an epithelial-like extracellular matrix, and stem cells with immunosuppression. Although few immune checkpoint was detected on cytotoxic immune cells such as $\mathrm{T}$ and NK cells, there was strong immunosuppression exerted by Tregs and M2 macrophages. In addition, the cellular interactions indicated an enhanced TGF- $\beta$ signaling pathway as the main mechanism for tumor progression, invasion, and immunosuppression in chordomas.

Conclusion: Our study contribute to the clarification of intra-tumoral heterogeneity, and may pave the way to identify potential therapeutic targets in chordomas.

\section{Introduction}

Chordoma is a rare, aggressive, and locally invasive bone tumor, and it arises from undifferentiated notochordal remnants. The incidence of chordoma is 0.08 per 100,000 people[1]. Chordomas occurs more commonly in older adults and are predominantly located in axial skeleton, with the most common sites being the sacrum (50\%-60\%), skull base (25\%-35\%), and spine (15\%) [2,3]. Chemotherapy is not effective against chordoma. The generally accepted treatment of chordoma is en bloc excision, and radiotherapy is recommended in subtotal or piecemeal excisions or in cases of recurrence. [2]. Many patients with chordomas, however, undergo very morbid surgeries and eventually die from tumor recurrence, with the 5- and 10-year survival rates were $67.6 \%$ and $39.9 \%$ respectively $[4,5]$. Thus, traditional treatment strategies have reached a limit in chordoma management [6]. Consequently, novel and effective therapies are needed for this rare but aggressive tumor.

Clinic studies of molecular targeted therapy (MTT) in chordomas have identified several potential therapeutic targets: PDGFR, EGFR, HER2, VEGFR, and others [7]. However, the therapeutic effect of these MTT drugs is limited, as the response rates of apatinib and sorafenib is only around $3.7 \%$. Moreover, the sorafenib has significant toxicity, with $77.8 \%$ grade 3 and $14.8 \%$ grade 4 toxicities noted in patients [8]. The immune check point inhibitors (ICls) have proven an effective treatment in other cancers such as sarcomas, but these favorable results are limited to tumors that are mismatch repair deficient [9]. However, programmed cell death-1 (PD-1) or programmed cell death-ligand 1 (PD-L1) inhibitors show 
clinical benefit in some patients. Tumor heterogeneity is the main reason for the diverse response to these drugs[10]. The complex tumor microenvironment (TME) is the reason for the high heterogeneity. TME is made up of various cellular populations such as cancer cells, vascular cells, fibroblasts, and immune cells [11]. The TME of chordomas consists of different immune cell populations, which include a variety of immune checkpoints [12] and vascular endothelial cells [13]. However, it is difficult for bulk gene analyses to provide an accurate reflection of the TME. [14]. Consequently, the heterogeneity of chordomas is not well studied, and novel methods are needed to accurately assess this heterogeneity.

Single-cell RNA sequencing (scRNA-seq) may provide elucidation of the mechanisms underlying carcinogenesis and of the molecular features of cancers. It may provide a clearer description of the TME at the single cell level and potentially allow for more effective therapeutic strategies for tumors [15]. scRNA-seq is currently used to delineate the complicated tumor heterogeneity in several malignancies such as glioblastomas [16], liver cancers [17] , lung cancers [18] , renal cell carcinoma [19] and head and neck cancers[20]. However, tumor heterogeneity and cellular interactions in chordomas are poorly understood. We used an scRNA-seq sequencing platform $(10 \times$ Genomics $)$ to describe the tumor landscape of chordomas in 6 patients. The goal was to explore tumor heterogeneity and identify potential therapeutic targets in chordomas.

\section{Materials And Methods}

\section{Chordoma samples}

Six patients who underwent a surgical treatment at Xuanwu Hospital, Capital Medical University from July, 2019 to April 2020 were included in this study. All patients were diagnosed with pathology as having classic chordomas according to the 2013 WHO classification of bone tumors [21] (Figure S1A). Immunohistochemistry was performed [22], showing that all chordoma cells had strongly positive TBXT (brachyury) expression (Figure S1B). The samples were obtained from fresh tumors during surgery, and written informed consents were obtained from all patients. The study was conducted after approval of Ethics Committee of Xuanwu hospital of Capital Medical University (No.2016033).

\section{sc-RNA Sequencing}

Single-cell transcriptomic sequencing was performed (Capitalbio Technology Corporation, http://www.capitalbiotech.com). Following the manufacture's instructions for Single Cell 30 Library and Gel Bead Kit V2 (10x Genomics), cell suspensions were loaded on a Chromium Single Cell Controller (10x Genomics, San Francisco, CA) to generate single-cell gel beads in emulsion. After Drop-seq droplet collection, CDNA amplification and sequencing library preparations were carried out exactly as described previously [17], and the libraries were sequenced on an Illumina HiSeq X Ten. The libraries from one batch of droplets were sequenced separately for Drop-seq data from chordoma cells.

\section{Cell filtration, clustering and downstream analysis}


Downstream analysis was done using the Seurat package (version 3.2.0) [23] in R software (version 4.0.2), which provides functions from preprocessing to clustering, dimension reduction, visualization and differential gene expression. In processing, raw gene expression matrices were imported and filtrated by the following standards: 1) cells expressing a minimum of 200 genes and genes expressed by a minimum of 30 cells; 2 ) cells with the mitochondrial percentage of less than 25\% (Figure S2A, B). 33,737 high quality cells remained for downstream analysis. Furthermore, the Seurat package was used to normalize and integrate expression data and to remove batch effect. The new integrated matrix was used to scale, run PCA, and visualize the landscape with UMAP.

\section{Cell type annotation}

We adopted 19 clusters and identify all markers of clusters using the Seurat package. Cell types were annotated to acknowledged biological cell types by canonical marker genes. The methods implemented the SingleR package [24] (version 1.3.6), the CellMarker website [25], and the infercnv package (version 1.5.0). The TBXT gene, a specific feature of chordoma, was also used to annotate the tumor cells.

\section{Functional enrichment analysis}

After the annotation of each cell type, we employed a functional enrichment analysis to differentially expressing genes between different clusters for Gene Ontology (GO) and KEGG (Kyoto Encyclopedia of Genes and Genomes). This analysis was performed to illustrate the biological processes and potential functions of different cells using the clusterProfiler package (version 3.17.0)[26] and the org.Hs.eg.db package (version 3.11.4). The $p$ value cutoffs of GO and KEGG were both 0.05 . The top ten terms of the results were visualized by bar plot or dot plot.

\section{Pseudotime}

Trajectory analysis was performed using the monocle package (version 2.17.0) [27]. We included tumor cells, mononuclear phagocytes and fibroblasts. We performed analysis of each group with the following parameters: lowerDetectionLimit $=0.5$, min_expr $=0.1$, num_cells_expressed $>=10$. For visualization, the plot_cell_trajectory function was used to plot the potential trajectory according to pseudotime, seurat clusters and the data.

\section{Cellular communications}

Cell-to-cell interactions were analyzed using the CellChat package (version 0.0.1)[28]. The majority of ligand-receptor interaction were mainly identified on the basis of the KEGG signaling pathway database and recent peer-reviewed experimental studies. The main steps of inference of intercellular communications are as follows: 1) identification of differentially expressed signaling genes, 2) calculation of an ensemble of average expression, 3) calculation of intercellular communication probability. 


\section{Results}

\section{The Overall Landscape of the Chordoma Tumor Samples}

Six tumor samples were included in the scRNA-seq profiles (Figure 1A). There were four females and two males, with ages ranging from 17 to 49 . The chordomas were located in the sacrum $(n=1)$, mobile spine $(n=3)$, and skull base $(n=2)$. The detailed characteristics of the six chordomas are presented in the Supplement Table 1. After strict filtering, we retained 33,737 cells in the final analysis. After normalization of gene expression and principal component analysis (PCA), these cells were divided into 18 clusters using the umap method (Figure 1B). The 18 clusters were equally distributed among the 6 samples, indicating less batch-corrected effect (Figure S3A). We further assigned these clusters to 7 known cell lineages, which were chordoma cells, mononuclear phagocytes, T/NK cells, fibroblasts, endothelial cells, $B$ cells and neutrophils (Figure 1B-D). The marker genes for the seven cellular populations are presented in Figure 1B, and the characteristic genes are shown in the Supplement table 2. There were $6392(47.6 \%)$ chordomas cells, 6945 (20.6\%) fibroblasts, 4734 (14.0\%) mononuclear phagocytes, 3944 (11.7\%) T/NK cells, $780(2.3 \%)$ B cells, $878(2.6 \%)$ neutrophils and 404 (1.2\%) endothelial cells. We did not perform a downstream analysis of the B cells, neutrophils and endothelial cells because of the small numbers. There was a varied proportion of cellular populations, indicating an inter-tumor heterogeneity with potential clinical significance (Figure 1E, Figure S3B, C). The primary tumor location was a strong prognostic factor and was related to the extent of surgical resection [29].

\section{Transcriptomic tumor heterogeneity of tumor cells in chordoma}

There were 16,052 tumor cells identified according to the chordoma-specific marker, TBXT. These chordomas cells were further assigned to 6 clusters with differently expressed genes (DEGs) (Figure 2 A, B). There was a high degree of tumor heterogeneity both within (Figure 2A) and between patients (Figure S4A). These six tumor subclusters also varied according the tumor location (Figure S4B). The GO analysis was performed to investigate the biological function of DEGs, which mainly participate in the immune response, including leukocyte migration, regulation, chemotaxis, regulation of B cell activation and the humoral immune response (Figure $2 \mathrm{C}$ ). These results suggest that the tumor cell's heterogeneity was the reason for the varied tumor immunologic microenvironment. We next analyzed the expression of major histocompatibility complex (MHC) class I genes and PD-L1/PD-L2 expression on chordoma cells

(Figure S4C). However, most tumor cells did not express MHC genes or PD-L1, and the results here discouraged the usage of PD-1 inhibitors for chordomas.

There were 6950 chordomas cells in subcluster 0 , and this was the largest tumor population (43.3\%). It was mainly involved in the extracellular matrix (ECM) organization by GO analysis (Figure S4D) and was characterized by CTGF, COL2A1, COL6A3 and COL5A2 (Table S3). We designed them as matrix tumor cells (mTCs, mTCs-C0-CTGF). Cluster 1 (4408 cells, 27.5\%) displayed a high expression of genes related to metal ion metabolism, such as MT1X, MT2A and MT1G. The enrichment analysis exhibited associated metal ion related pathways (Figure S4D), and we named them epithelial tumor cells (eTCs-C1IGFBP3). Cluster 4 was involved in nuclear division (Figure S4D), which implied active tumor cell 
proliferation. The biomarkers for cancer stem cells (CSCs) were expressed in this cluster. Such biomarkers as STMN1 [31, 32], UBE2C [33] and PTTG1[34] [35] were associated with tumor cell survival, clonality and tumorigenicity in several malignancies. This subcluster was regarded as stem tumor cells (sTCs, mTCs-C4-STMN1). The pseudo time analysis showed subcluster 4 was in the early stage of tumor cells. The deficiency of SMARCB1 chordoma indicated a malignant subtype and was associated with a poor prognosis [36, 37]. We found that clusters 2, 3, and 5 were devoid of SMARCB1 (Figure S4D). Neither these three clusters expressed CDKN2A, which was frequent lossed in chordomas [38] (Figure S4E). Next, we conducted a pseudo time analysis of 6 clusters to investigate their developmental trajectories, and we found that clusters 2, 3 and 5 tended to be in a more advanced stage (Figure 2D, Figure S4F). Interestingly, there were studies indicating a close relationship between SMARCB1 loss and tumor immune microenvironment changes [39]. The subclusters 2 and 5 devoid of SMARCB1 was involved in immune functions, which is consistent with the immune-related effect of SMARCB1 in chordomas (Figure S4D). Lastly, we analyzed the MTT target expression in chordomas, which were EGFR, HER2, PDGFR, VEGF, VEGFR2, and stem cell factor receptor (KIT) (Figure 2E). Itt showed that VEGF and VEGFR2 were most upregulated in chordomas, indicating potential therapeutic targets.

\section{T/NK cell clustering and subtype analysis}

The unsupervised clustering was performed for 3944 T/NK cell populations; this was divided into 5 subclusters with their unique signature genes (Figure 3A, B). Cluster 2 was identified as NK cells (374 cells, 9.5\%) marked with KRF1, and others were T cells (3570 cells, 90.5\%) with CD3E expression (Figure 3C). It showed a high heterogeneity of tumor associated with T/NK cells among patients (Figure S5A), but an association with tumor location (Figure S5B). The T cells were annotated as follows. The C0, C1, C3 and C4 were identified as CD4 ${ }^{+} \mathrm{T}$ (2298 cells, $\left.64.4 \%\right), \mathrm{CD}^{+} \mathrm{T}$ (953 cells, $26.7 \%$ ), CD4 CD8 double negative (160 cells, $4.5 \%)$, and Treg cells (159 cells, $4.4 \%$ ) respectively. The specific makers for the immune cells identified above were CD4, CD8 and foxp3 respectively (Figure $3 C$ ). Notably, we found a cluster of double negative $T$ cells which were involved in the activity of systemic inflammation, tissue damage and tumor [40]. There were only 160 DNT cells detected in chordomas, and these were not included in the downstream analysis. The $\mathrm{C}^{-} \mathrm{CD} 8^{+} \mathrm{T}$ and $\mathrm{C2}^{-} \mathrm{NK}^{+}$showed a high level of cytotoxic activity which was characterized by GZMA, GZMK, GNLY, PRF1 and IFNY (Figure 3B, Table S4). Moreover, there were few immune checkpoints expressed on these cytotoxic immune cells (Figure 3C). Interestingly, there was increased CTLA4, TIGIT and TIM3 expression on Tregs (Figure 3D), which serve as main immunosuppressive mechanisms and therapeutic targets for chordomas.

\section{Mononuclear phagocyte clustering and subtype analysis}

To intestigate the heterogeneity among macrophages, 3,698 tumor-associated with macrophages (TAMs) were clustered into six subgroups (Figure 4A): C0 (1414 cells, 29.9\%), C1 (1061 cells, 22.4\%), C2 (807 cells, $17.0 \%)$, C3 (743 cells, 15.7\%), C4 (367 cells, 7.8\%), and C5 (342 cells, 7.2\%). The C0 and C1 represented the largest cellular populations, and they were associated with $\mathrm{M} 2$ subtype polarization because they exhibited high levels CD68+CD163+CD204+ expression (Figure 4B). In addition, it was 
shown that VEGFA was heavily expressed in these cells from cluster 1 (Table S3). The M2-subtype TAMs secrete VEGFA for promoting tumor growth[41]. Our GO analysis also found that this cluster was involved in the regulation of angiogenesis (Figure $4 \mathrm{C}$ ). The sub-cluster 1 is marked with inflammatory chemokines such as CCL3, CXCL2, and CXCL3 (Table S5), consistent with the GO analysis (Figure 4C). Release of these proteins by TAMs may promote an inflammatory response and tumor development. The data showed that cluster 0 and 1 represented an M2-like TAM cluster. Moreover, the genes S100A8, S100A9, and S100A12, which encode calcium-binding proteins, were expressed by the cells in cluster 2 (Table S3) [42]. Activated mononuclear cells can release these proteins, which has been shown to promote inflammatory responses in vivo. Thus, these data indicate that C3 may have a pro-inflammatory and antitumor role in in chordomas. C4 and C5 indicate nuclear division and neutrophil activation, respectively (Figure 4C). Lastly, we found that CD47 and TIM3 were expressed on TAMs (Figure 4D); however, other immune checkpoints were not (data not shown).

\section{Distinct fibroblast subpopulations in human chordomas}

There were 6945 fibroblast cells identified with the specific markers, and these cells were further divided into 6 subclusters (Figure 5A). All six subclusters expressed high levels of canonical fibroblast markers such as ACTA2 (a-SMA), COL1a2, and PDGFRb, confirming their identity as fibroblasts (Figure 5C). We found that these subclusters exhibited distinct transcriptomic signatures (Figure 5B, Table S6). There were 2600 cells in the subcluster 0 , accounting for the largest population (37.4\%) of the fibroblast cells. The sub-clusters were characterized by ECM signatures, which included collagen molecules (COL1A1, COL3A1, COL4A1), POSTN, and LUM. Also, the GO analysis for this subtype was related to ECM and collagen fibril organization (Figure 5D). We designated them as matrix CAFs (mCAFs, mCAFs-C0POSTN; Figure 5B, C). The sub-cluster 1 consisted of 1185 fibroblast cells and was the second largest population (17.1\%). The significant genes up-regulated in this subtype were IL1RL1, CCL3, CCL4I2, CCL8 and others (Table S6). The GO analysis confirmed this subtype was involved in cell chemotaxis and T cell activation, and we named it inflammatory CAFs (iCAFs, iCAFs-C1-IL1RL1; Figure 5B, C). The C2 subcluster consisted of 910 fibroblast cells, and marked with CRABP2, which was associated with osteogenic differentiation. The $\mathrm{GO}$ analysis found that it was involved in ossification and cartilage development; therefore, we regarded it as bone-development regulation CAFs (bCAFs, bCAFs-c2-IL1RL1; Figure. 5B, C). 796 fibroblasts were identified in the sub-cluster 3, and the marked genes were CST1, ANGPTL4. Moreover, the $\mathrm{GO}$ analysis of this $\mathrm{C} 3$ was related to regulation of vasculature development. The $\mathrm{C} 3$ was then regarded as vascular CAFs (vCAFs, vCAFs-c3-MCAM; Figure. 5B, C). However, we found a similar function of $\mathrm{C} 5$ as $\mathrm{C} 3$, as the marker genes of $\mathrm{C} 5$ were characterized by microvasculature signature genes (MCAM, MYH11, GJA4, and RGS5). Subcluster 5 fibroblasts mainly expressed epithelium-specific marker genes such as KRT19 and KRT8, which we designated as EMT-like CAFs (eCAFs, eCAFs-c5-KRT19; Figure 5B, C).

The TGF $\beta$ signaling pathway was enriched by the interplay between $\mathrm{CD} 4^{+} \mathrm{T}$ cells, fibroblasts, and macrophages. 
We first analyzed the overall cellular interactions based on ligand-receptor pairs, and we found that the TGF $\beta$ signaling pathway was most active in the TME of chordomas (Figure 6A, Figure S6). TGFB1TGFBR1/TGFBR2 and TGFB1-ACVR1/TGFBR1 were the dominant contributors to this communication network (Figure 6D), which was consistent with previous reports that TGFB1 was a potent TGF $\beta$ ligand in cancers [43]. Moreover, the TGF $\beta$ signaling played a key role in tumor progression by regulating different effects on multiple cell types, which included malignant and non-cancerous cells within the TME [44]. This showed that TGF $\beta$ was mainly produced and released by fibroblasts and macrophages in chordomas (Figure 6B). However, this TGF $\beta$ signaling pathway network was strongly active among macrophages, fibroblast cells, tumor cells and $\mathrm{CD} 4^{+} \mathrm{T}$ cells (Figure $6 \mathrm{C}$ ). Although a small population of Tregs was detected, they expressed a number of immune checkpoints, which exerted strong immunosuppressive effects (Figure 3D). The TGF $\beta$ signaling pathway was one main reason for the transformation of $\mathrm{CD}^{+} \mathrm{T}$ cells into Tregs in the TME of chordomas. Interestingly, the cytotoxic immune cells such as $C D 8^{+} T$ cells and NK cells did not express immune checkpoints, resulting in no effect on TGF $\beta$ (Figure 3D) [45, 46]. Lastly the chordomas cells exhibited properties of ECM, epithelial cells, and stem cells (Figure S4D). The enhanced TGF $\beta$ signaling was associated with malignant biological processes of ECM, tumor stem cell properties, and epithelial-to-mesenchymal transition (EMT) [47-49]. Consequencently, the malignant cells were mainly influenced by TGF $\beta$ pathway in chordomas.

\section{Discussion}

In this study, we first delineated the transcriptomic landscape of chordomas by single-cell RNA sequencing. The cellular populations of TME in chordomas included tumor cells, fibroblast and immune cells; this varied within and between tumors, indicating a high tumor heterogeneity. Moreover, there was a variety of tumor heterogeneity according to tumor location, suggesting novel mechanisms for different clinical outcomes based upon chordoma location. Molecular therapy targets and immunotherapy biomarkers were also analyzed in this study, and these provided potential valuable therapeutic targets. The types of cellular interactions indicated enhanced the TGF $\beta$ signaling pathway was a main mechanism for tumor progression and invasion and immunosuppression in chordomas. Our study first investigated the tumor heterogeneity at the single cell level, hopefully providing new insight into mechanism for tumor development and for possible therapeutic strategies for chordomas.

The chordomas cells accounted for approximately half of the total cellular populations, and were made up of 6 subclusters. These subclusters were involved in malignant cell properties such as ECM, epithelial like, stem cell and immunosuppression. There were 4408 (27.5\% of total tumor cells) chordomas cells in subcluster 1 which were related to epithelial cells. Previous studies have suggested a dual epithelialmesenchymal differentiation of chordomas [50], and these subclusters were likely to resist chemotherapy and radiotherapy for chordomas. The biomarkers (CD133 ${ }^{+}, \mathrm{CD}_{15}{ }^{+}, \mathrm{WNT} 5, \mathrm{ABTG} 2$ and MYCBP) of CSCs were identified in chordomas, [51], but these makers were not detected here. Instead, the biomarkers of CSCs such as STMN1, UB2C and PTTG1 in hepatocellular carcinoma, breast cancer, prostate tumors were detected here [31-35]. The pathological mechanisms of these markers should be further investigated 
in the future studies. In addtion, our results found that VEGF and VEGFR-2 were the most upregulated in chordomas. These results are consist with the clinical evidence that sorafenib and apatinib have an objective response for chordomas.[7]

Our results showed a complicated immune TME of chordomas which was made up by macrophages, $T$ and NK cells. The $C D 8^{+} T$ cells and NK cells showed a high cytotoxic activity, characterized by GZMA, GZMK, GNLY, PRF1 and IFNy. Also there two cytotoxic immune cells were devoid of immune checkpoints. However, there was a strong immunosuppressive mechanism noted, which was mainly exerted by Tregs and macrophage. CTLA4, TIGIT and TIM3 were heavily expressed on Tregs in our study. Also Tregs were detected in human chordoma samples and associated with poor clinical outcomes by strongly suppressing immune function $[52,53]$. Our results also showed that tumor associated with macrophages (TAMs) mainly exhibited the M2-subtype, which is involved in angiogenesis and immunosuppression. As CD47 and TIM3 are heavily expressed on the TAMs, immunotherapy may reverse the negative role of TAMs in chordomas.

The cellular interactions identified the TGF $\beta$ signaling pathway as a key role in tumor development, tumor invasion, immunosuppression and CSCs properties. The fibroblast and macrophage were the main source of TGF $\beta$ in chordomas according to previous reports [54-56]. The chordomas cells may strongly be influenced by TGF $\beta$ in a variety of malignant biological factors such as ECM, CSCs properties and epithelial like characteristics $[57,58]$. Moreover, the TGF $\beta$ signaling pathway also had a negative effect on immune cells, which were $\mathrm{CD} 4^{+} \mathrm{T}$ cells. The $\mathrm{CD} 4^{+} \mathrm{T}$ cells were found to be the largest immune cell population in chordomas. The $\mathrm{CD} 4^{+} \mathrm{T}$ cells may be transformed into Tregs or Th0 cells under the influence of certain cytokines or chemokines [59]. The $C D 4^{+} T$ cells were affected by the TGF $\beta$ signaling pathway and evolved into Tregs in our study. Together, these results indicate that the TGF $\beta$ signaling pathway is a fundamental mechanism for tumor development and immunosuppression in chordomas.

\section{Conclusion}

In conclusion, our findings provide a large transcriptomic landscape of chordomas to the detail of singlecell resolution and provide an established resource for elucidating chordoma diversity. However, the detailed mechanisms and efficacy of therapeutics still needed to be further explored in experimental and clinical studies.

\section{Abbreviations}

MTT: molecular targeted therapy

ICls: check point inhibitors

PD-1: programmed cell death-1

PD-L1: programmed cell death-ligand 1 
TME: tumor microenvironment

scRNA-seq: Single-cell RNA sequencing

GO: Gene Ontology

KEGG: Kyoto Encyclopedia of Genes and Genomes

DEGs: differently expressed genes

MHC: major histocompatibility complex

ECM: extracellular matrix

mTCs: matrix tumor cells

eTCs: epithelial tumor cells

CSCs: cancer stem cells

sTCs: stem tumor cells

TAMs: tumor-associated with macrophages

EMT: epithelial-to-mesenchymal transition

\section{Declarations}

\section{Availability of data and material}

The raw sequencing data that support the findings of this study were deposited to the Genome Sequence Archive of Beijing Institute of Genomics, Chinese Academy of Sciences: HRA000513 (http://bigd.big.ac.cn/gsa-human/browse/HRA000513).

\section{Acknowledgements}

We acknowledge the contributions of specific colleagues, institutions, or agencies that aided the efforts of the authors.

\section{Funding}

CAMS/PUMC Research Project \#201920200501, Human Brain Tissue Bank Platform for Neurological Diseases

\section{Author information}

Affiliations: 
Department of Neurosurgery, Xuanwu Hospital, Capital Medical Universiy. Beijing, China

Wanru Duan, Boyan Zhang, Shanhang Jia, Zong Xin, Qing Jian ,Fengzeng Jian \&Zan Chen

Lab of Spinal Cord Injury and Functional Reconstruction, China International Neuroscience Institute (CHINA-INI)

Wanru Duan, Fengzeng Jian \&Zan Chen

Department of Neurological Surgery, University of California San Francisco, San Francisco, California, USA

Dean Chou

Department of liver transplantation, The Affiliated Hospital of Qingdao University, Qingdao, China

Xinqiang Li

Contributions :

Wan-ru Duan, Shan-hang Jia: conception, design .Bo-yan Zhang , Zong Xin: collection and assembly of data, data analysis and interpretation. All authors: manuscript writing, final approval of manuscript and accountable for all aspects of the work.

Corresponding:

Correspondence to Zan Chen.

\section{Ethics declarations}

Ethics approval and consent to participate:

The study was conducted after approval of Ethics Committee of Xuanwu hospital of Capital Medical University (No.2016033).

Consent for publication:

The study was conducted after consent for publication from patients.

Competing interests:

The authors declared no conflict of interest.

\section{References}


1. McMaster ML, Goldstein AM, Bromley CM, Ishibe N, Parry DM: Chordoma: incidence and survival patterns in the United States, 1973-1995. Cancer causes \& control : CCC 2001, 12(1):1-11.

2. Walcott BP, Nahed BV, Mohyeldin A, Coumans JV, Kahle KT, Ferreira MJ: Chordoma: current concepts, management, and future directions. The Lancet Oncology 2012, 13(2):e69-76.

3. Chugh R, Tawbi H, Lucas DR, Biermann JS, Schuetze SM, Baker LH: Chordoma: the nonsarcoma primary bone tumor. Oncologist 2007, 12(11):1344-1350.

4. Tarpey PS, Behjati S, Young MD, Martincorena I, Alexandrov LB, Farndon SJ, Guzzo C, Hardy C, Latimer C, Butler AP et al: The driver landscape of sporadic chordoma. Nat Commun 2017, 8(1):890.

5. Thanindratarn P, Dean DC, Nelson SD, Hornicek FJ, Duan Z: Advances in immune checkpoint inhibitors for bone sarcoma therapy. Journal of bone oncology 2019, 15:100221.

6. Stacchiotti S, Sommer J, Chordoma Global Consensus G: Building a global consensus approach to chordoma: a position paper from the medical and patient community. Lancet Oncol 2015, 16(2):e7183.

7. Meng T, Jin J, Jiang C, Huang R, Yin H, Song D, Cheng L: Molecular Targeted Therapy in the Treatment of Chordoma: A Systematic Review. Frontiers in oncology 2019, 9:30.

8. Bompas E, Le Cesne A, Tresch-Bruneel E, Lebellec L, Laurence V, Collard O, Saada-Bouzid E, Isambert N, Blay JY, Amela EY et al: Sorafenib in patients with locally advanced and metastatic chordomas: a phase II trial of the French Sarcoma Group (GSF/GETO). Annals of oncology : official journal of the European Society for Medical Oncology 2015, 26(10):2168-2173.

9. Tawbi HA, Burgess M, Bolejack V, Van Tine BA, Schuetze SM, Hu J, D'Angelo S, Attia S, Riedel RF, Priebat DA et al: Pembrolizumab in advanced soft-tissue sarcoma and bone sarcoma (SARC028): a multicentre, two-cohort, single-arm, open-label, phase 2 trial. The Lancet Oncology 2017, 18(11):1493-1501.

10. Guo M, Peng Y, Gao A, Du C, Herman JG: Epigenetic heterogeneity in cancer. Biomarker research 2019, 7:23.

11. Potter SS: Single-cell RNA sequencing for the study of development, physiology and disease. Nat Rev Nephrol 2018, 14(8):479-492.

12. Zou MX, Lv GH, Wang XB, Huang W, Li J, Jiang Y, She XL: Clinical Impact of the Immune Microenvironment in Spinal Chordoma: Immunoscore as an Independent Favorable Prognostic Factor. Neurosurgery 2019, 84(6):E318-e333.

13. Morimoto Y, Tamura R, Ohara K, Kosugi K, Oishi Y, Kuranari Y, Yoshida K, Toda M: Prognostic significance of VEGF receptors expression on the tumor cells in skull base chordoma. 2019, 144(1):65-77.

14. Wang Y, Mashock M, Tong Z, Mu X, Chen H, Zhou X, Zhang H, Zhao G, Liu B, Li X: Changing Technologies of RNA Sequencing and Their Applications in Clinical Oncology. Front Onco/ 2020, 10:447.

15. Zhang Y, Zhang Z: The history and advances in cancer immunotherapy: understanding the characteristics of tumor-infiltrating immune cells and their therapeutic implications. Cellular \& 
molecular immunology 2020, 17(8):807-821.

16. Neftel C, Laffy J, Filbin MG, Hara T, Shore ME, Rahme GJ, Richman AR, Silverbush D, Shaw ML, Hebert CM et al: An Integrative Model of Cellular States, Plasticity, and Genetics for Glioblastoma. Cell 2019, 178(4):835-849.e821.

17. Zhang M, Yang H, Wan L, Wang Z, Wang H, Ge C, Liu Y, Hao Y, Zhang D, Shi G et al: Single-cell transcriptomic architecture and intercellular crosstalk of human intrahepatic cholangiocarcinoma. Journal of hepatology 2020, 73(5):1118-1130.

18. Maynard A, McCoach CE, Rotow JK, Harris L, Haderk F, Kerr DL, Yu EA, Schenk EL, Tan W, Zee A et al: Therapy-Induced Evolution of Human Lung Cancer Revealed by Single-Cell RNA Sequencing. Cell 2020, 182(5):1232-1251.e1222.

19. Pan XW, Zhang H, Xu D, Chen JX, Chen WJ, Gan SS, Qu FJ, Chu CM, Cao JW, Fan YH et al: Identification of a novel cancer stem cell subpopulation that promotes progression of human fatal renal cell carcinoma by single-cell RNA-seq analysis. International journal of biological sciences 2020, 16(16):3149-3162.

20. Puram SV, Tirosh I, Parikh AS, Patel AP, Yizhak K, Gillespie S, Rodman C, Luo CL, Mroz EA, Emerick KS et al: Single-Cell Transcriptomic Analysis of Primary and Metastatic Tumor Ecosystems in Head and Neck Cancer. Cell 2017, 171(7):1611-1624.e1624.

21. Jo VY, Fletcher CD: WHO classification of soft tissue tumours: an update based on the 2013 (4th) edition. Pathology 2014, 46(2):95-104.

22. Wang LM, Song C, Li YX, Zhang XD, Ji YH, Wen WJ: A novel isocitrate dehydrogenase 1 G131D mutation in glioblastoma. Chinese medical journal 2020.

23. Stuart T, Butler A, Hoffman P, Hafemeister C, Papalexi E, Mauck WM, 3rd, Hao Y, Stoeckius M, Smibert P, Satija R: Comprehensive Integration of Single-Cell Data. Cell 2019, 177(7):1888-1902 e1821.

24. Aran D, Looney AP, Liu L, Wu E, Fong V, Hsu A, Chak S, Naikawadi RP, Wolters PJ, Abate AR et al: Reference-based analysis of lung single-cell sequencing reveals a transitional profibrotic macrophage. Nature immunology 2019, 20(2):163-172.

25. Zhang X, Lan Y, Xu J, Quan F, Zhao E, Deng C, Luo T, Xu L, Liao G, Yan M et al: CellMarker: a manually curated resource of cell markers in human and mouse. Nucleic acids research 2019, 47(D1):D721D728.

26. Yu G, Wang LG, Han Y, He QY: clusterProfiler: an R package for comparing biological themes among gene clusters. Omics : a journal of integrative biology 2012, 16(5):284-287.

27. Trapnell C, Cacchiarelli D, Grimsby J, Pokharel P, Li S, Morse M, Lennon NJ, Livak KJ, Mikkelsen TS, Rinn JL: The dynamics and regulators of cell fate decisions are revealed by pseudotemporal ordering of single cells. Nature biotechnology 2014, 32(4):381-386.

28. Jin S, Guerrero-Juarez CF, Zhang L, Chang I, Myung P, Plikus MV, Nie Q: Inference and analysis of cellcell communication using CellChat. bioRxiv 2020:2020.2007.2021.214387.

29. Zheng W, Huang Y, Guan T, Lu S, Yao L, Wu S, Chen H, Wang N, Liang Y, Xiao W et al: Application of nomograms to predict overall and cancer-specific survival in patients with chordoma. Journal of 
bone oncology 2019, 18:100247.

30. Meng T, Jin J, Jiang C, Huang R, Yin H, Song D, Cheng L: Molecular Targeted Therapy in the Treatment of Chordoma: A Systematic Review. Frontiers in oncology 2019, 9(30).

31. Zhang R, Gao X, Zuo J, Hu B, Yang J, Zhao J: STMN1 upregulation mediates hepatocellular carcinoma and hepatic stellate cell crosstalk to aggravate cancer by triggering the MET pathway. 2020, 111(2):406-417.

32. Obayashi S, Horiguchi J, Higuchi T, Katayama A, Handa T, Altan B, Bai T, Bao P, Bao H, Yokobori T et al: Stathmin 1 expression is associated with aggressive phenotypes and cancer stem cell marker expression in breast cancer patients. International journal of oncology 2017, 51(3):781-790.

33. Shuliang S, Lei C, Guangwu J, Changjie L: Involvement of ubiquitin-conjugating enzyme E2C in proliferation and invasion of prostate carcinoma cells. Oncology research 2013, 21(3):121-127.

34. Parte S, Virant-Klun I, Patankar M, Batra SK, Straughn A, Kakar SS: PTTG1: a Unique Regulator of Stem/Cancer Stem Cells in the Ovary and Ovarian Cancer. Stem cell reviews and reports 2019, 15(6):866-879.

35. Huang S, Liu Q, Liao Q, Wu Q, Sun B, Yang Z, Hu X, Tan M, Li L: Interleukin-6/signal transducer and activator of transcription 3 promotes prostate cancer resistance to androgen deprivation therapy via regulating pituitary tumor transforming gene 1 expression. 2018, 109(3):678-687.

36. Hasselblatt M, Thomas C, Hovestadt V, Schrimpf D, Johann P, Bens S, Oyen F, Peetz-Dienhart S, Crede Y, Wefers A et al: Poorly differentiated chordoma with SMARCB1/INI1 loss: a distinct molecular entity with dismal prognosis. Acta neuropathologica 2016, 132(1):149-151.

37. Shih AR, Cote GM, Chebib I, Choy E, DeLaney T, Deshpande V, Hornicek FJ, Miao R, Schwab JH, Nielsen GP et al: Clinicopathologic characteristics of poorly differentiated chordoma. Modern pathology : an official journal of the United States and Canadian Academy of Pathology, Inc 2018, 31(8):1237-1245.

38. Cottone L, Eden N, Usher I, Lombard P, Ye H, Ligammari L, Lindsay D, Brandner S, Pižem J, Pillay N et al: Frequent alterations in p16/CDKN2A identified by immunohistochemistry and FISH in chordoma. $J$ Pathol Clin Res 2020, 6(2):113-123.

39. Gounder MM, Zhu G, Roshal L, Lis E, Daigle SR, Blakemore SJ, Michaud NR, Hameed M, Hollmann TJ: Immunologic Correlates of the Abscopal Effect in a SMARCB1/INI1-negative Poorly Differentiated Chordoma after EZH2 Inhibition and Radiotherapy. Clinical cancer research : an official journal of the American Association for Cancer Research 2019, 25(7):2064-2071.

40. Brandt D, Hedrich CM: TCRalphabeta(+)CD3(+)CD4(-)CD8(-) (double negative) $T$ cells in autoimmunity. Autoimmunity reviews 2018, 17(4):422-430.

41. Hwang I, Kim JW, Ylaya K, Chung EJ, Kitano H, Perry C, Hanaoka J, Fukuoka J, Chung JY: Tumorassociated macrophage, angiogenesis and lymphangiogenesis markers predict prognosis of nonsmall cell lung cancer patients. 2020, 18(1):443.

42. Heilmann RM, Nestler J, Schwarz J, Grützner N, Ambrus A, Seeger J, Suchodolski JS, Steiner JM, Gurtner C: Mucosal expression of S100A12 (calgranulin C) and S100A8/A9 (calprotectin) and 
correlation with serum and fecal concentrations in dogs with chronic inflammatory enteropathy. Veterinary immunology and immunopathology 2019, 211:64-74.

43. Nüchel J, Ghatak S, Zuk AV, Illerhaus A, Mörgelin M, Schönborn K, Blumbach K, Wickström SA, Krieg T, Sengle $G$ et al: TGFB1 is secreted through an unconventional pathway dependent on the autophagic machinery and cytoskeletal regulators. Autophagy 2018, 14(3):465-486.

44. Derynck R, Turley SJ, Akhurst RJ: TGF $\beta$ biology in cancer progression and immunotherapy. 2020.

45. Takeuchi M, Kosiewicz MM, Alard P, Streilein JW: On the mechanisms by which transforming growth factor-beta 2 alters antigen-presenting abilities of macrophages on $\mathrm{T}$ cell activation. European journal of immunology 1997, 27(7):1648-1656.

46. Kobie JJ, Wu RS, Kurt RA, Lou S, Adelman MK, Whitesell LJ, Ramanathapuram LV, Arteaga CL, Akporiaye ET: Transforming growth factor beta inhibits the antigen-presenting functions and antitumor activity of dendritic cell vaccines. Cancer research 2003, 63(8):1860-1864.

47. Lamouille S, Xu J, Derynck R: Molecular mechanisms of epithelial-mesenchymal transition. Nature reviews Molecular cell biology 2014, 15(3):178-196.

48. Miettinen PJ, Ebner R, Lopez AR, Derynck R: TGF-beta induced transdifferentiation of mammary epithelial cells to mesenchymal cells: involvement of type I receptors. The Journal of cell biology 1994, 127(6 Pt 2):2021-2036.

49. Kornberg TB: Distributing signaling proteins in space and time: the province of cytonemes. Current opinion in genetics \& development 2017, 45:22-27.

50. Gulluoglu S, Turksoy O, Kuskucu A, Ture U, Bayrak OF: The molecular aspects of chordoma. Neurosurgical review 2016, 39(2):185-196; discussion 196.

51. Tuysuz EC, Gulluoglu S, Yaltirik CK, Ozbey U, Kuskucu A, Çoban EA, Sahin F, Türe U, Bayrak OF: Distinctive role of dysregulated miRNAs in chordoma cancer stem-like cell maintenance. Experimental cell research 2019, 380(1):9-19.

52. Zou MX, Guo KM, Lv GH, Huang W, Li J, Wang XB, Jiang Y, She XL: Clinicopathologic implications of CD8(+)/Foxp3(+) ratio and miR-574-3p/PD-L1 axis in spinal chordoma patients. Cancer immunology, immunotherapy : Cll 2018, 67(2):209-224.

53. Zou MX, Zheng BW, Liu FS, Wang XB, Hu JR, Huang W, Dai ZH, Zhang QS, Liu FB, Zhong H et al: The Relationship Between Tumor-Stroma Ratio, the Immune Microenvironment, and Survival in Patients With Spinal Chordoma. Neurosurgery 2019, 85(6):E1095-e1110.

54. Hildenbrand R, Jansen C, Wolf G, Böhme B, Berger S, von Minckwitz G, Hörlin A, Kaufmann M, Stutte $\mathrm{HJ}$ : Transforming growth factor-beta stimulates urokinase expression in tumor-associated macrophages of the breast. Laboratory investigation; a journal of technical methods and pathology 1998, 78(1):59-71.

55. Wei Y, Kim TJ, Peng DH, Duan D, Gibbons DL, Yamauchi M, Jackson JR, Le Saux CJ, Calhoun C, Peters $\mathrm{J}$ et al: Fibroblast-specific inhibition of TGF- $\beta 1$ signaling attenuates lung and tumor fibrosis. The Journal of clinical investigation 2017, 127(10):3675-3688. 
56. Correction: Tumor-Secreted LOXL2 Activates Fibroblasts through FAK Signaling. Molecular cancer research : MCR 2019, 17(10):2141.

57. Scheel C, Weinberg RA: Cancer stem cells and epithelial-mesenchymal transition: concepts and molecular links. Seminars in cancer biology 2012, 22(5-6):396-403.

58. Ramachandran A, Vizán P, Das D, Chakravarty P, Vogt J, Rogers KW: TGF- $\beta$ uses a novel mode of receptor activation to phosphorylate SMAD1/5 and induce epithelial-to-mesenchymal transition. 2018, 7.

59. Stadhouders R, Lubberts E, Hendriks RW: A cellular and molecular view of T helper 17 cell plasticity in autoimmunity. Journal of autoimmunity 2018, 87:1-15.

\section{Figures}


A

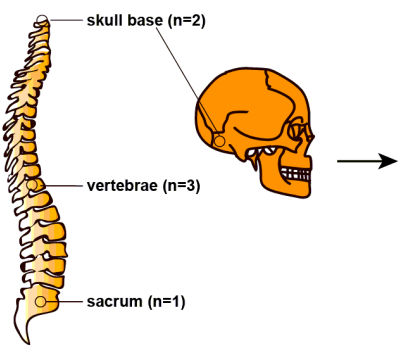

Surgical resection samples

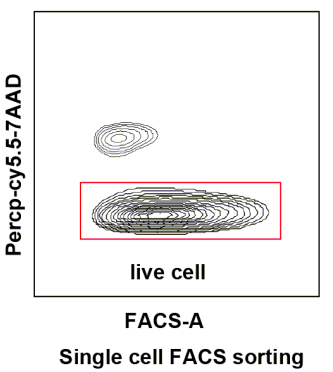

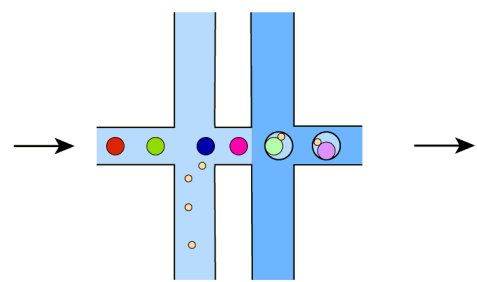

10X genomics mRNA sequencing

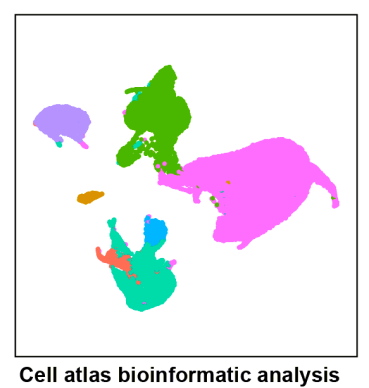

Cell atlas bioinformatic analysis
B

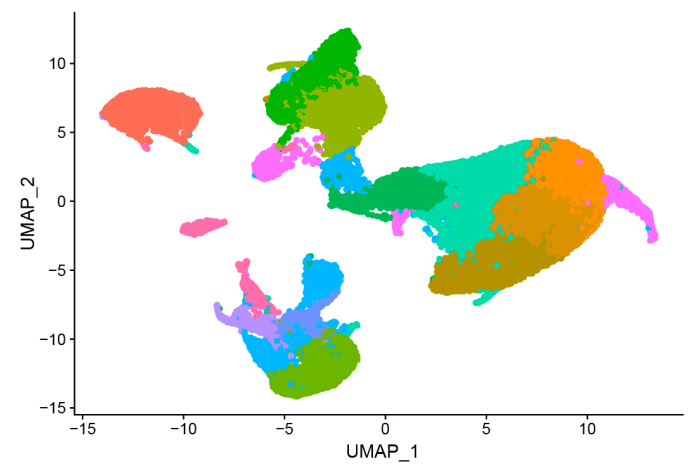

C
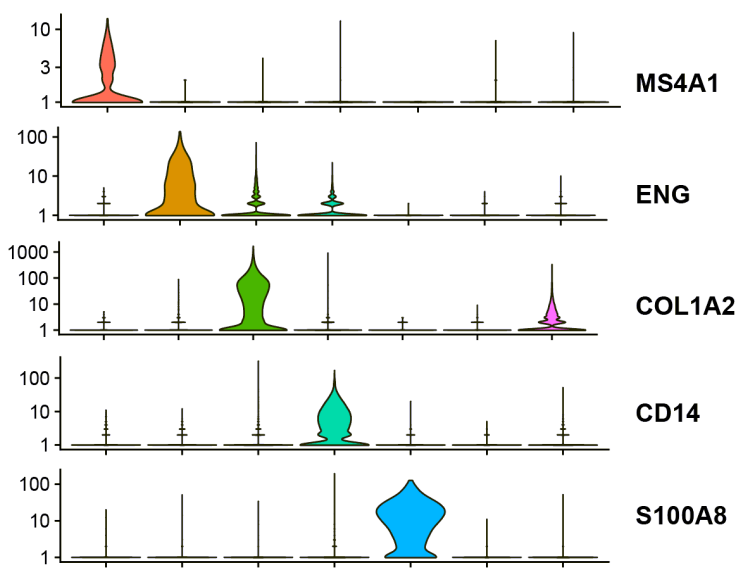

S100A8

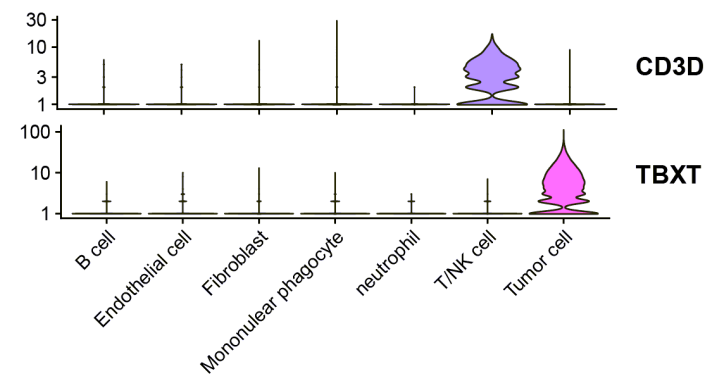

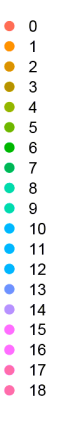

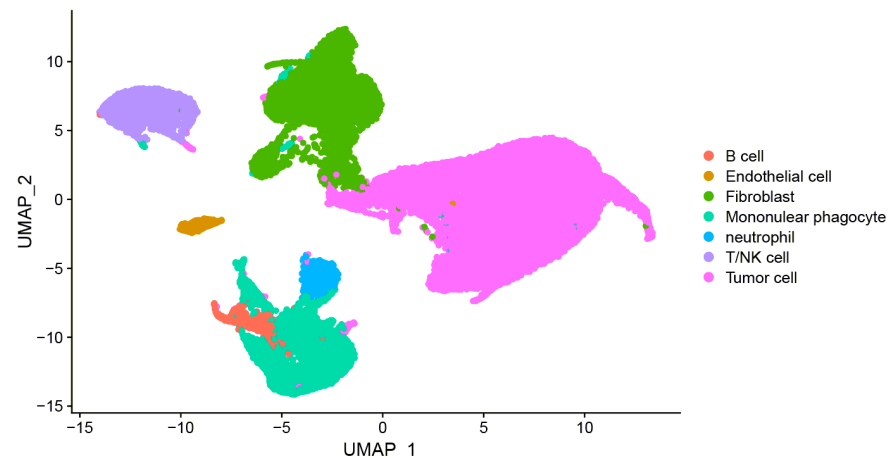

\section{D}

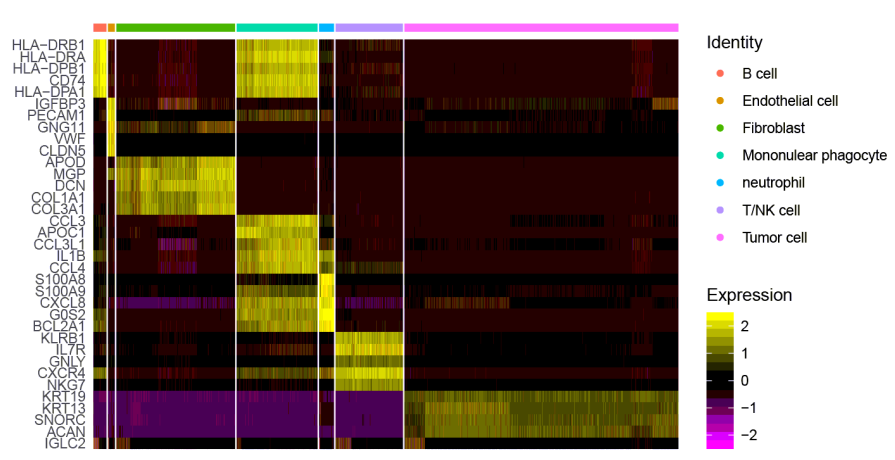

$E$

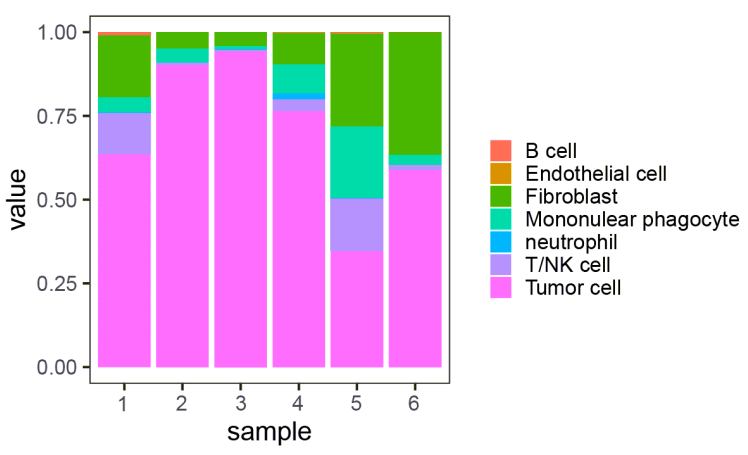

\section{Figure 1}

Overall Landscape of Chordoma tumor sample (A) Schematic diagram of scRNA-seq analysis workflow. (B) UMAP clustering for 33737cells and the cell type identification. (C) Marker genes for seven distinct cell types. (D) The heatmap of the top five DEGs of each cell type. (E) The proportion of identified cell type in samples. 
A

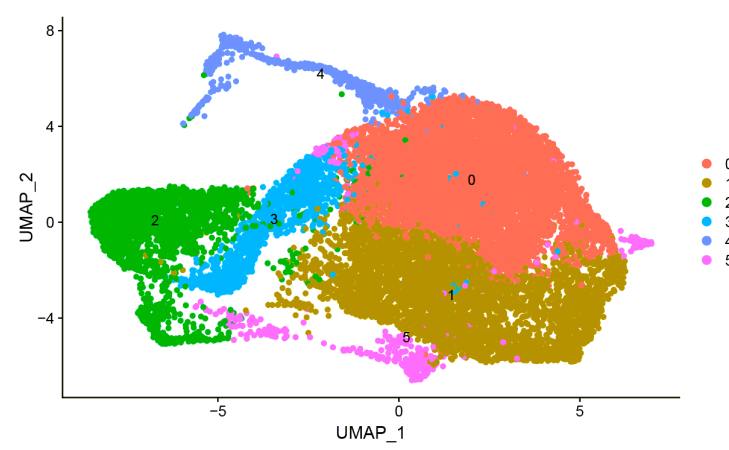

C

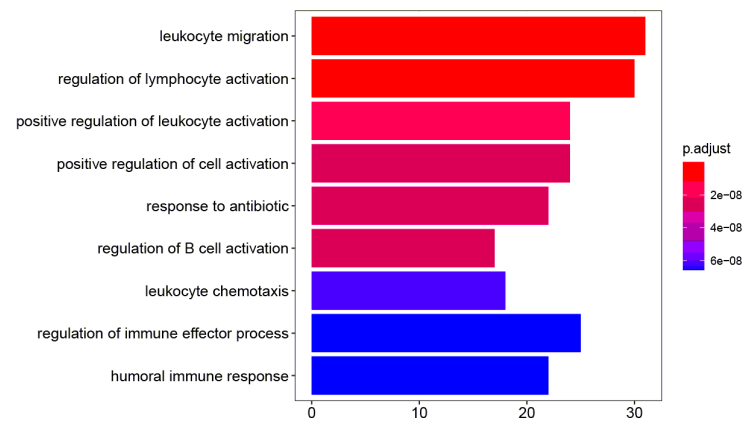

$E$

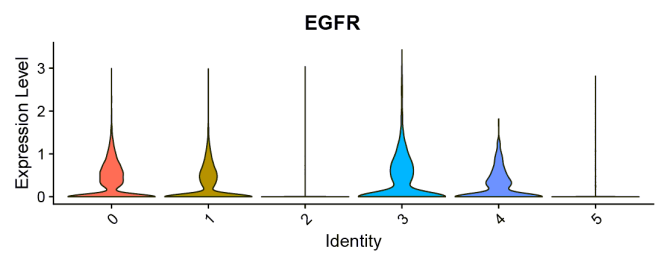

KIT

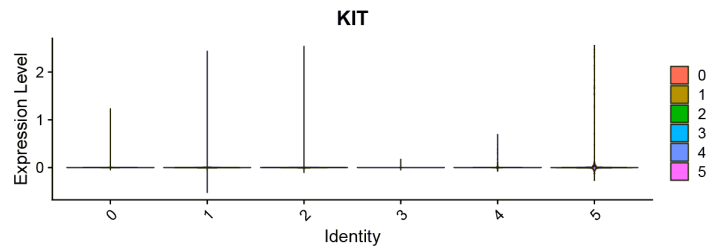

VEGFA

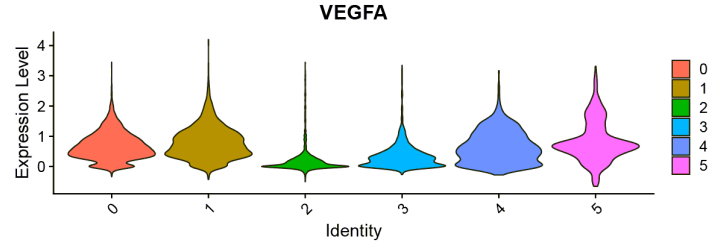

B

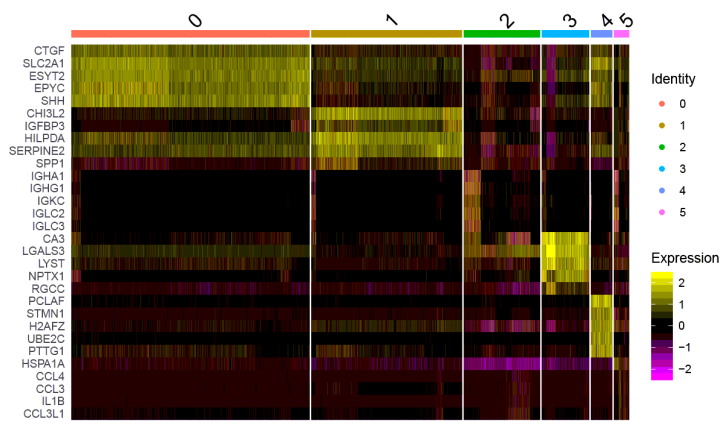

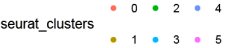
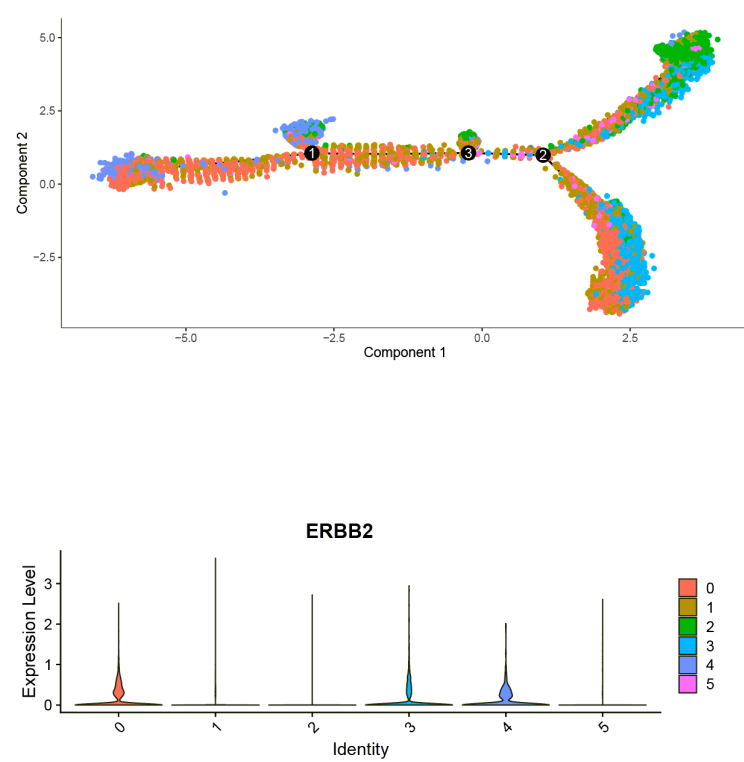

PDGFRB

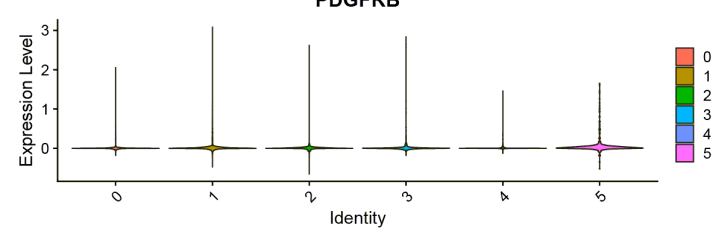

KDR

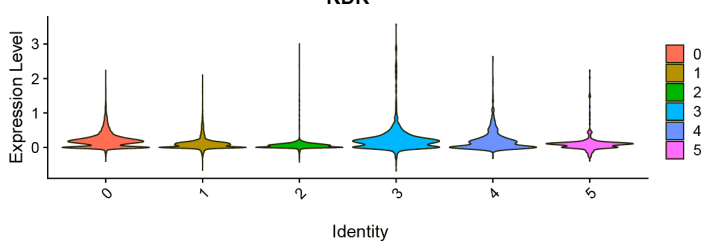

\section{Figure 2}

Transcriptomic tumor heterogeneity of tumor cells in chordoma (A) Sub clustering for tumor cells. (B) Heatmap of the top five DEGs. (C) GO terms for DEGs (D) The pseudotime analysis of tumor cells. (E) MTT targets expression in each cluster. 
A

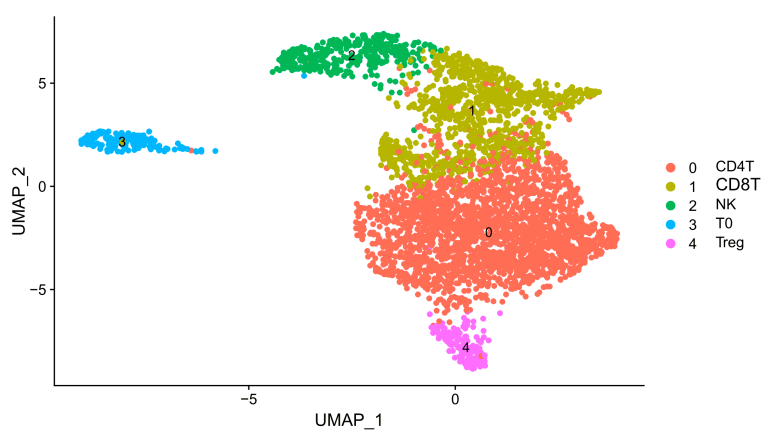

C
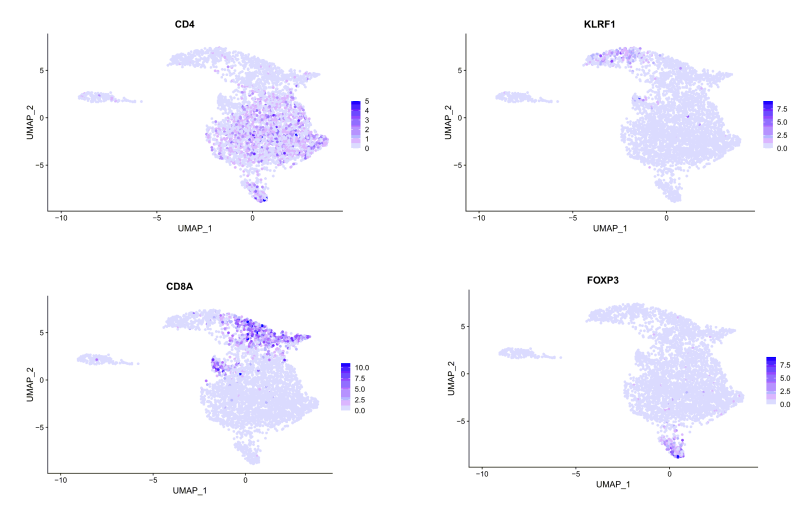

B

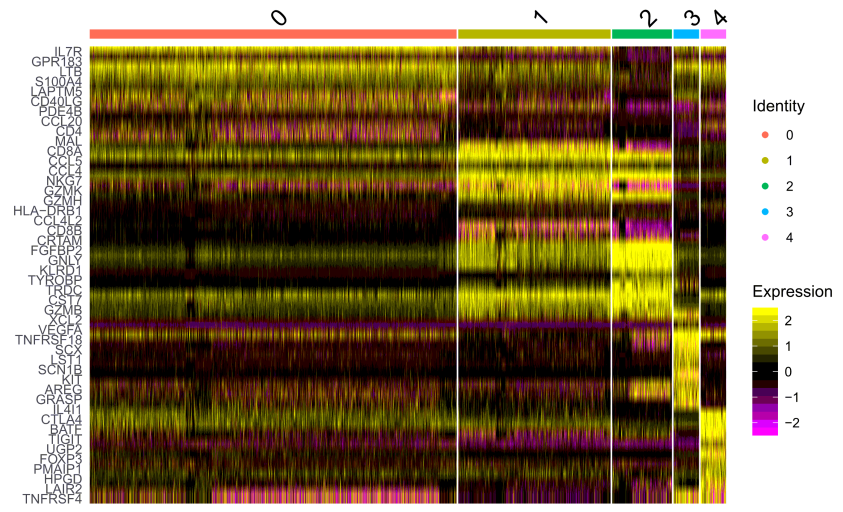

D
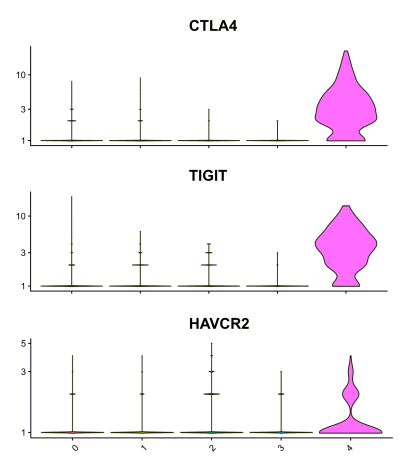
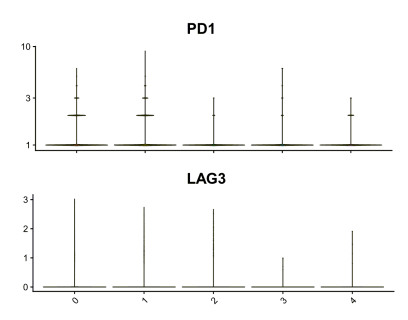

PDL1

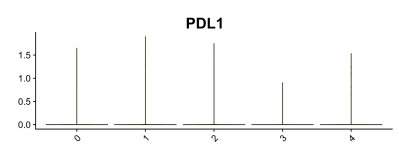

\section{Figure 3}

T, NK cells clustering and subtype analysis (A) Clustering for T cells and NK cells. (B) Heatmap of the top 10 DEGs of subclusters of T/NK cells. (C) expression of marker genes for each cell type. (D) The expression of the immune checkpoints. 
A

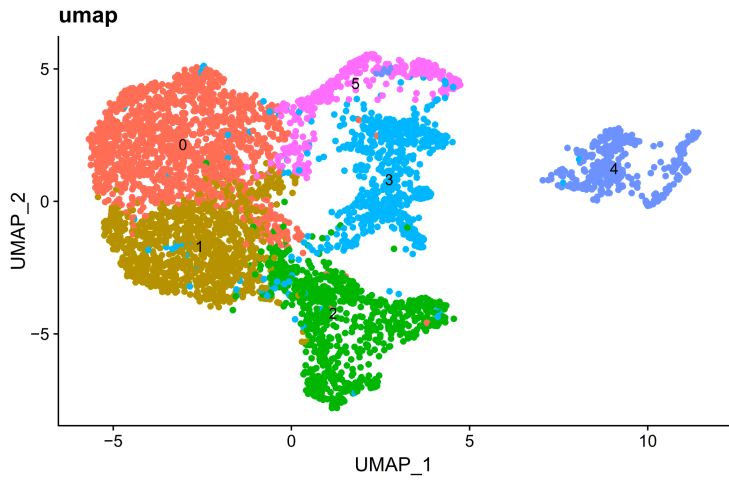

C
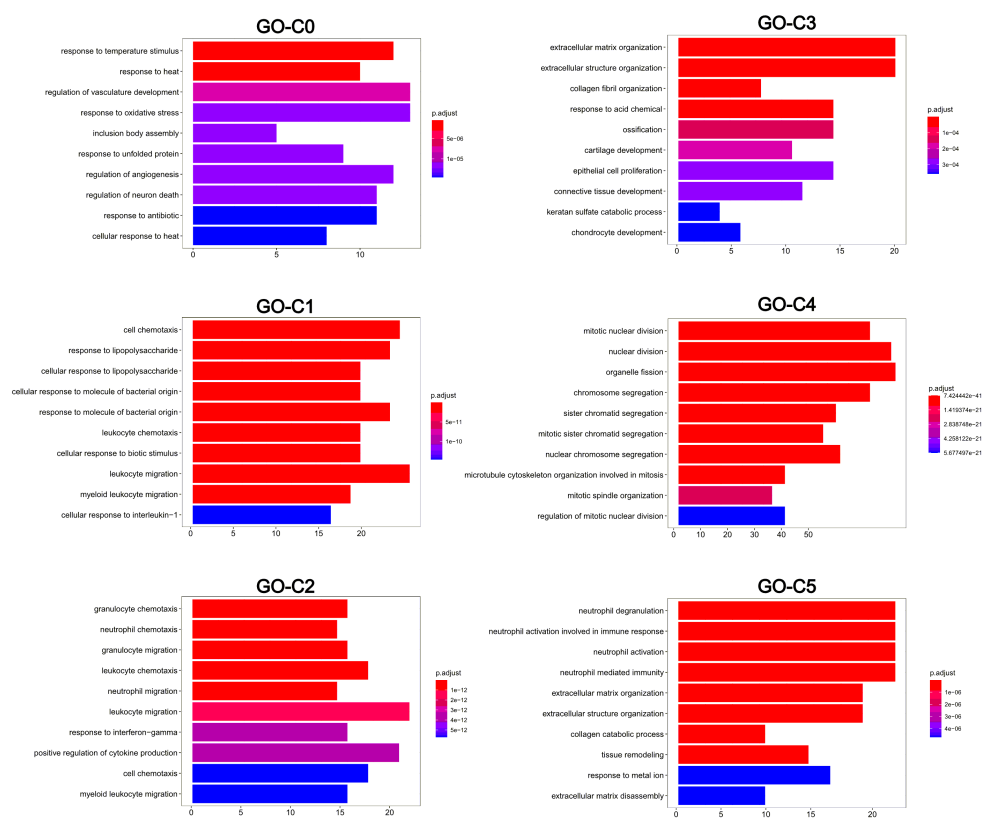

B

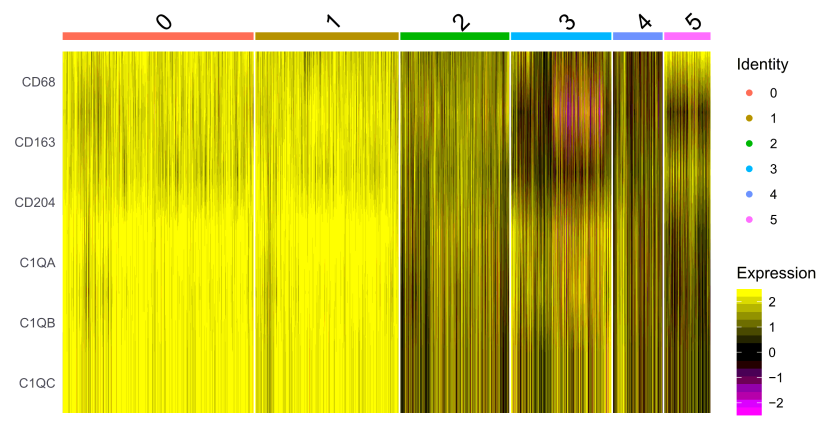

D

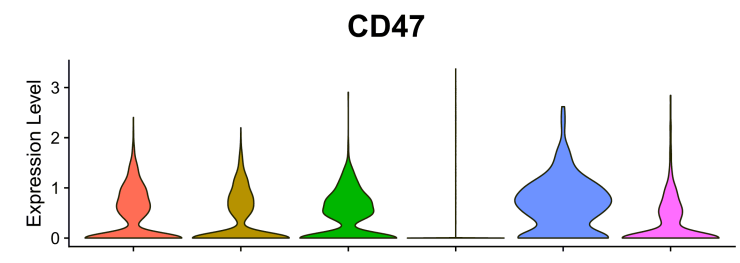

HAVCR2

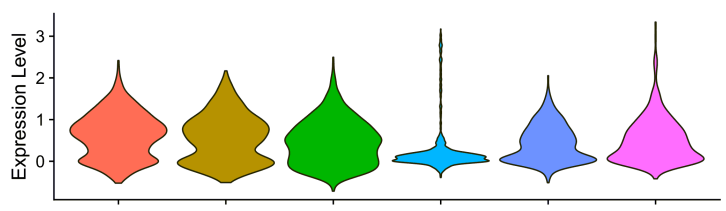

CTLA4

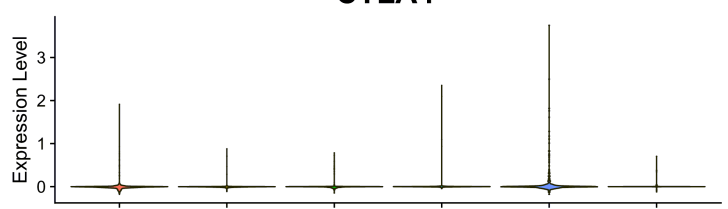

\section{Figure 4}

Mononuclear phagocytes clustering and subtype analysis (A) Subclustering for mononuclear phagocytes. (B) Biomarkers for M2-TMAs expression. (C) GO analysis for subclusters. (D) Violin plots showing the expression of CD47 and HAVCR2. 
A

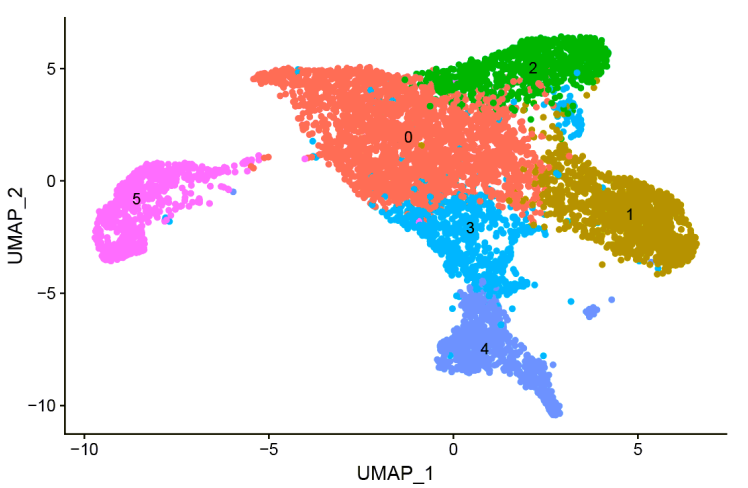

C

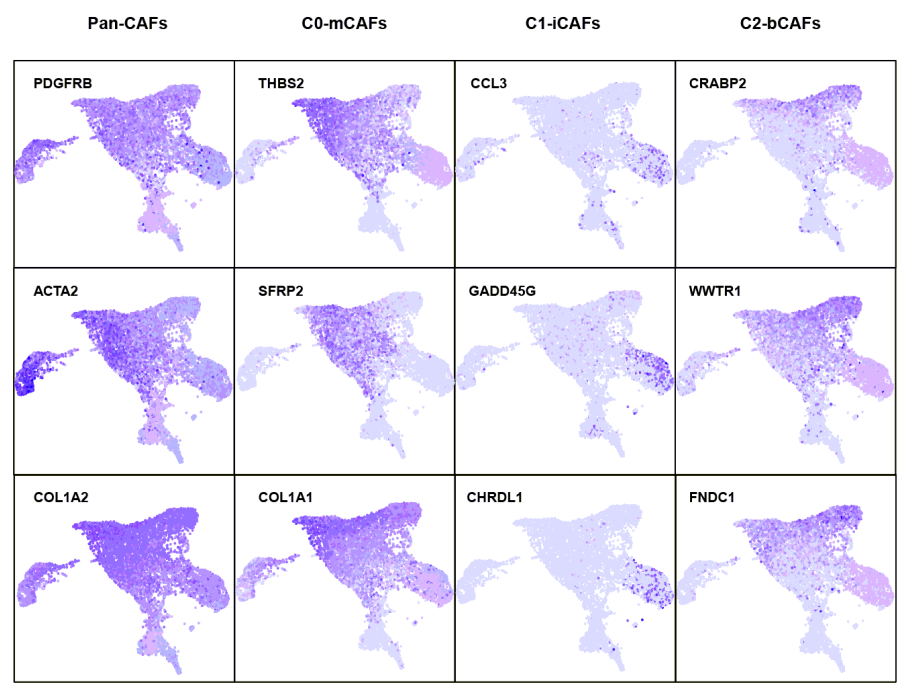

B

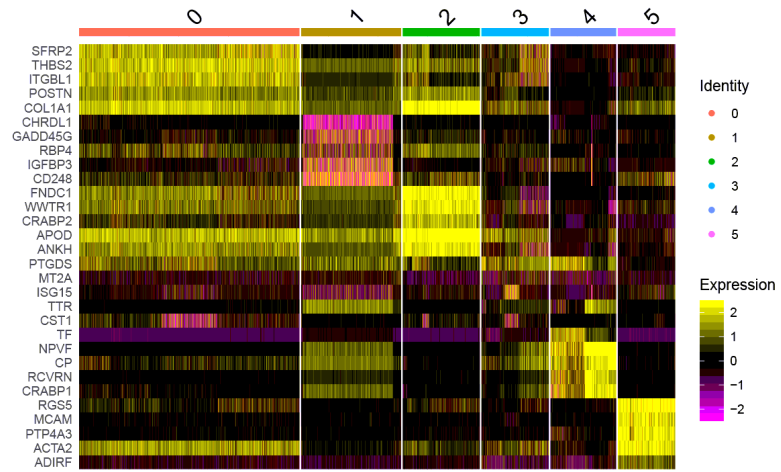

D
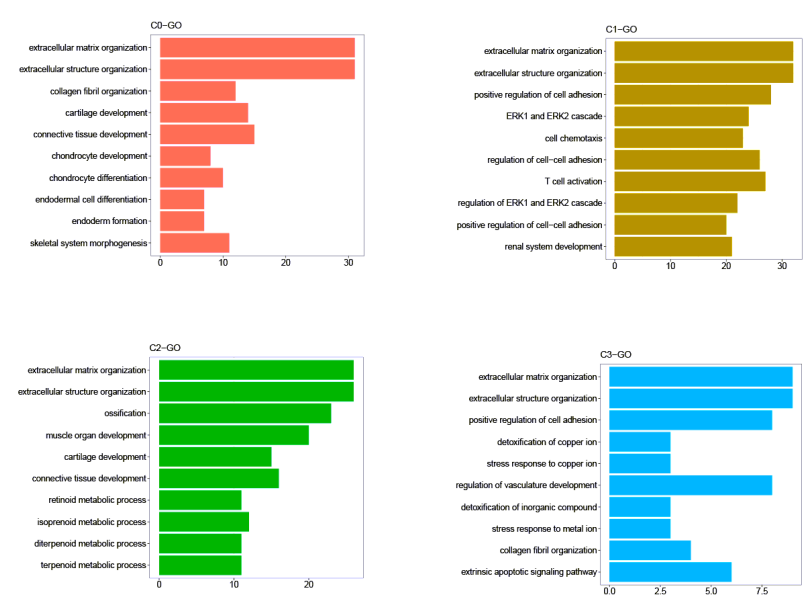

\section{Figure 5}

Distinct fibroblast subpopulations in human chordomas (A) UMAP plots of total fibroblasts color-coded for 6 clusters. (B) Heatmap expression showing the top five DEGs of each cluster. (C) UMAP plots colorcoded for the expression of marker genes for distinct cell subclusters. (D) GO analysis of DEGs in distinct fibroblast clusters. 
A
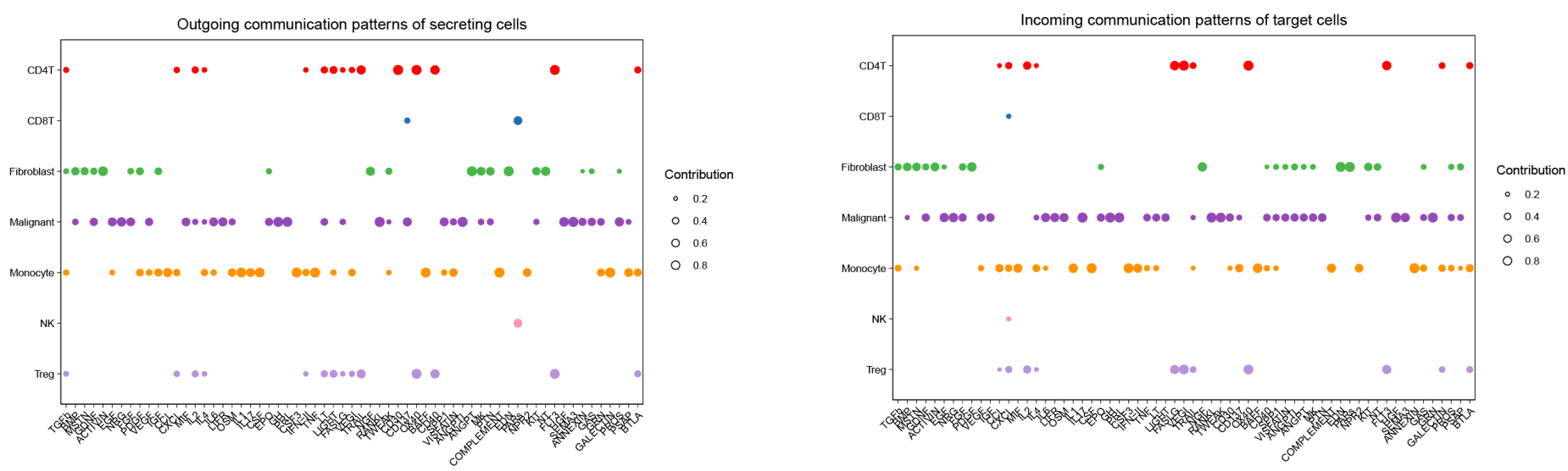

B

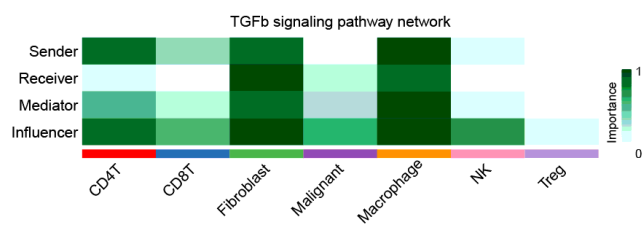

$\mathrm{D}$

C
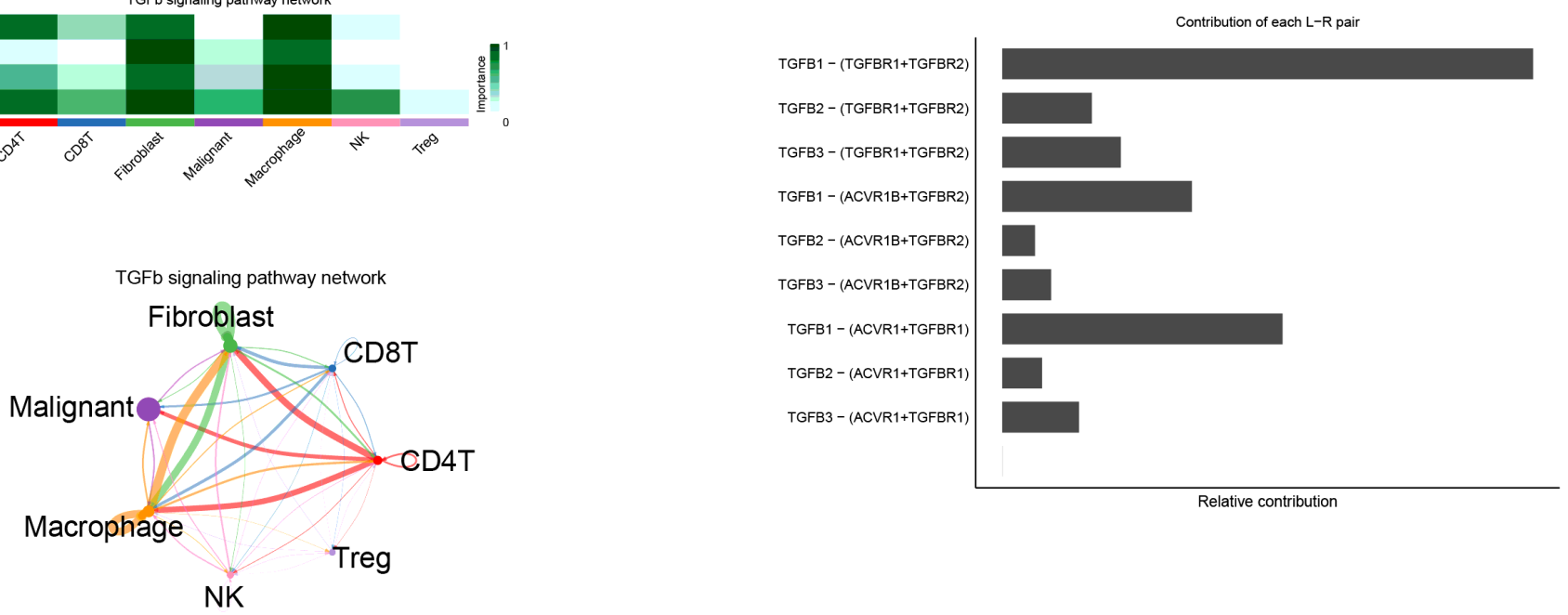

Figure 6

The TGF $\beta$ signaling pathway was enriched in the interplay between CD4+ T cells, fibroblast, and macrophage. (A) Dot plots showing the outgoing and incoming communication patterns of the whole distinct cell types. (B) Heatmap of the relative importance of each cell group based on the computed four network centrality measures of TGF $\beta$ signaling. (C) Circle plot showing the inferred TGF $\beta$ signaling networks. (D) Relative contribution of each ligand-receptor pair to the overall communication network of TGF $\beta$ signaling pathway.

\section{Supplementary Files}

This is a list of supplementary files associated with this preprint. Click to download.

- FigureS1.pdf

- Figures2.tif

- FigureS3.tif

- FigureS4.tif 
- Figures5.tif

- Figures6.tif

- Tables1.xIsx

- Tables2total.csv

- Tables3tumor.csv

- TableS4TNK.csv

- TableS6Fibroblast.csv 